\title{
Early sonographic markers and prediction of pregnancy outcome: a prospective study
}

\author{
Jaishree Bamniya*, Divyesh Panchal, Pooja Singh, Ami Shah, Harshad Ladola
}

Department of Obstetrics and Gynecology, GCS Medical College, Ahmedabad, Gujrat, India

Received: 05 March 2017

Revised: 16 April 2017

Accepted: 17 April 2017

\author{
*Correspondence: \\ Dr. Jaishree Bamniya, \\ E-mail: sh1311@yahoo.in, jaishreekumar@yahoo.in
}

Copyright: ( $)$ the author(s), publisher and licensee Medip Academy. This is an open-access article distributed under the terms of the Creative Commons Attribution Non-Commercial License, which permits unrestricted non-commercial use, distribution, and reproduction in any medium, provided the original work is properly cited.

\begin{abstract}
Background: The objective of the study was to examines the pregnancy outcome in patients with early sonography markers and study design was a prospective study.

Methods: This prospective study was conducted at Department of Obstetrics and Gynaecology, GCS Medical College, Ahmedabad, Gujarat, India from Feb 2012 to August 2016. Total of 612 Women with early pregnancy were included in the study that fulfilled the inclusion criterias. After first sonography examination patients were assigned to the control group and study group. Patients were followed up for outcome.14 pateients were lost to follow up.

Results: The incidence of pregnancy loss was highest with Large yolk sac i.e 78.57\%, followed by loss with Bradycardia (38\%), Tachycardia (15.78\%) and Smaller than normal G Sac to be $14.28 \%$. As compared to control which had pregnancy loss of only $7.7 \%$. Incidence of other complications like IUGR, PIH were also increased in patients with abnormal early sonography markers up to $33 \%$.

Conclusions: $T$ Transvaginal sonography should be used adequately to look for early pregnancy markers like Size of Gestational sac, size and shape of yolk sac, fetal heart rate and in cases of threatened abortion presence of subchorionic haematoma and its size because all these markers are good predictors of pragnacy outcome and can prove helpful in patient counseling.
\end{abstract}

Keywords: Bradycardia, Gestational sac, IUGR, TVS, Yolk sac

\section{INTRODUCTION}

The early first trimester of pregnancy is the fragile period for pregnancy viability, with up to $50 \%$ of embryos being lost before the pregnancy is clinically detected, and up to $15 \%$ to $25 \%$ of clinical pregnancies spontaneously aborting. ${ }^{1}$

During the embryonic period, all of the main viscera are formed. In the fetal period, these structures grow and complete their functional development. Sonography is the pivotal examination in the evaluation of pregnancy. The first demonstration of an early intrauterine pregnancy by means of transvaginal ultrasound (TVS) was reported by Kratochwil. Ultrasound has not only changed the medical approach to spontaneous miscarriage but also the patient's perception of normal and abnormal fetal development in utero.

Using TVS, one of the first signs of an IUP is a Hypoechoic complex within the thickened Decidualized endometrium followed by appearance of yolk sac. The secondary yolk sac is the first extraembryonic structure that becomes sonographically visible within the gestational sac. During embryonic development, the yolk sac is the primary route of exchange between the embryo and the mother. The yolk sac also provides nutritional, immunologic, metabolic, endocrine, and hematopoietic functions until the placental circulation is established. ${ }^{2}$ Normally, the yolk sac appears as a circular structure 
with an anechoic center surrounded by a uniform welldefined echogenic wall. Therefore, the yolk sac is crucial in early embryonic life followed by appearance of embryo and cardiac activity. ${ }^{3}$

Normal FHR is $110 \mathrm{bpm}$ (mean) by 5-6 weeks and 170 bpm by $9-10$ weeks. This is followed by a decrease in FHR becoming on average: $\sim 150$ bpm by 14 weeks, 140 bpm by 20 weeksand. $130 \mathrm{bpm}$ by term. A slow fetal heart rate is termed a fetal bradycardia and is usually defined as: FHR $<100$ bpm before 6.3 weeks gestation, or FHR $<120$ bpm between 6.3 and 7.0 weeks. A rapid fetal heart rate is termed a fetal tachycardia and is usually defined as: FHR >160-180 bpm and FHR around 170 bpm may be classified as borderline fetal tachycardia. ${ }^{4}$

It is estimated that approximately $30 \%$ to $40 \%$ of implanted pregnancies result in spontaneous abortion during the first trimester, most of which occur in the very early stage. ${ }^{5}$ However, after demonstration of positive embryonic heart activity, the rate of spontaneous abortion declines to $2 \%$ to $5 \% .{ }^{6}$ The embryonic heartbeat can usually be identified by prenatal sonography at 6 weeks' gestation with a transvaginal transducer, and the heart rate can be evaluated via $\mathrm{M}$-mode sonography. Previous studies have found association between severe embryonic bradycardia and fetal loss. ${ }^{7,8}$

By the appearance of these structures and their measurement we can predict the outcome of pregnancy to a large extent. Numerous sonographic signs of predictors of poor outcome have been described by various authors, including an excessively large, excessively small, or irregularly shaped gestational sac, a low implantation site, a large or irregular yolk sac, a weak decidual reaction, and a slow embryonic heart rate. ${ }^{9-12}$

\section{METHODS}

\section{Study design}

This was a prospective study. Institutional ethical Committee's and departmental review board's approval was taken for this study. Informed consent was obtained before the enrollment.

\section{Study setting and population}

This prospective study was conducted at Department of Obstetrics and Gynecology, GCS Medical College, Ahmedabad, Gujarat, India from Feb 2012 to August 2016. All pregnant women in early first trimester of pregnancy were included in the study who fulfilled the inclusion criteria. After taking the consent, Evaluation of patients was done which included Obstetrical history, clinical examination, relevant investigation and Lastly Ultrasonic examination which included after early scan all study subjects were scheduled for 11-14 weeks' scan, 20 weeks scan to rule out any structural abnormality. Followed by follow up scan at 28, 34, and 38 weeks for growth and as and when necessary to look for IUGR, oligohydramnios and abnormal doppler findings. Same patients were followed up till delivery to find out fetal outcome. 14 patients were lost to follow up during the study period. Logiq-3 Sonography Machine from GE was used for sonographic evaluation. Transvaginal sonography was done with the real-time sector scanner using high-frequency endovaginal probe $(5 / 7.5 \mathrm{MHz})$, after the patient voids urine. Transabdominal ultrasound scanning was done with real time scanners with low frequency probe $(3 / 3.5 \mathrm{MHz})$.

\section{Inclusion criteria}

- All pregnant patients who present in our Antenatal Clinic at 6- 8 weeks of gestation.

- Pregnant patients with history of bleeding per vaginum during present pregnancy.

- Pregnant patients with Abnormal Sonographic Markers Eg. Large or calcified yolk sac, Slow Embroynic Heart Rate, Small or Irregular Gestational Sac, Retrochorionic or Retroplacental collection.

\section{Exclusion criteria}

- Patients presenting with Extrauterine Pregnancy and Multiple pregnancy.

- Patients diagnosed with Missed Abortion on First visit to our Antenatal clinic.

- Patients who want to terminate the pregnancy.

- Patients who are known case of Chronic Hypertension, Patients who are on Antiepileptic, Antipsychiatric drugs.

Table 1: Outcome of pregnancies with abnormal heart rate.

\begin{tabular}{|llll|}
\hline Abortion & Bradycardia $(\mathbf{n = 2 1})$ & Tachycardia $(\mathbf{n = 1 9})$ & Control $(\mathbf{n = 4 1 2})$ \\
\hline Abruption & $8(38 \%)$ & $3(15.78)$ & $32(7.7 \%)$ \\
\hline IUGR (early onset /late onset) & - & - & $6(1.4 \%)$ \\
\hline PIH & $7(33.33 \%)$ & $10(47.61 \%)$ & $41(9.9 \%)$ \\
\hline Normal outcome & $5(23.80 \%)$ & $5(26.31 \%)$ & $35(8.4 \%)$ \\
\hline
\end{tabular}




\section{RESULTS}

In present study, we concluded that patients with abnormal early sonographic markers especially Large yolk sac and Tachycardia showed higher incidence of complications like Abortion, IUGR, Abruption and Pregnancy induced hypertension.
In present study, patients who presented with bleeding and had subchorionic haematoma $<4 \mathrm{~cm}^{2}$ had less incidence of spontaneous abortion $(16.66 \%)$ as compared to $25.69 \%$ in group with haematoma $>4 \mathrm{~cm}^{2}$. The patients in Group ' $A$ ' had better chances of Term delivery with supportive treatment i.e. $39.5 \%$ as compared to $3.4 \%$ in Group 'B'.

Table 2: Outcome of pregnancies with abnormal sized gestational sac.

\begin{tabular}{|lllll|}
\hline & Small GS $(\mathrm{n}=14)$ & Large GS $(\mathrm{n}=2)$ & Control $(\mathrm{n}=412)$ & Control (n=297) normal GS \\
\hline Abortion & $2(14.28 \%)$ & - & $32(7.7 \%)$ & - \\
\hline $\begin{array}{l}\text { Abruption } \\
\text { IUGR (early onset /late } \\
\text { onset) }\end{array}$ & $2(14.28 \%)$ & - & $6(1.4 \%)$ & - \\
\hline PIH & $4(28.57 \%)$ & - & $41(9.9 \%)$ & - \\
\hline Normal outcome & - & - & $35(8.4 \%)$ & - \\
\hline
\end{tabular}

Table 3: Outcome of pregnancies with large yolk sac.

\begin{tabular}{|lll|}
\hline & $\begin{array}{c}\text { Large yolk } \\
\text { sacs }(\mathbf{n}=14)\end{array}$ & Control $(\mathbf{n = 4 1 2})$ \\
\hline Abortion & $11(78.57 \%)$ & $32(7.7 \%)$ \\
\hline Abruption & - & $6(1.4 \%)$ \\
\hline $\begin{array}{l}\text { IUGR (early } \\
\text { onset /late onset) }\end{array}$ & $3(21.42 \%)$ & $41(9.9 \%)$ \\
\hline PIH & - & $35(8.4 \%)$ \\
\hline Normal outcome & - & $298(72.33 \%)$ \\
\hline
\end{tabular}

In present study, patients with history of threatened abortion especially the patients who had bleeding which was excessive or for prolonged duration had maximum chances of IUGR (34.7\%) followed by Preterm PROM $(5.5 \%)$ and preterm delivery $(4.5 \%)$.

Table 4: Size of sub-chorionic haemorrhage and pregnancy outcome $(n=144)$.

\begin{tabular}{|lll|} 
& $\begin{array}{l}<4 \mathrm{~cm}^{2} \\
(\text { Group A) }\end{array}$ & $\begin{array}{l}>4 \mathrm{~cm}^{2} \\
(\text { Group B) }\end{array}$ \\
\hline Spontaneous Abortion & $24(16.66 \%)$ & $37(25.69 \%)$ \\
\hline Preterm delivery & $15(10.41 \%)$ & $6(4.5 \%)$ \\
\hline Term delivery & $57(39.58 \%)$ & $5(3.4 \%)$ \\
\hline
\end{tabular}

\section{DISCUSSION}

In our study, we found that pregnant women with large gestational sac had normal fetal and maternal outcome while patients with small gestational sac had more incidence of missed or spontaneous abortion (14.28\%) and abruption placentae (14.28\%) while controls with normal growth of gestational sac had $7.7 \%$ and $1.4 \%$ abortion and abruption respectively. So, it showed that small gestatinal sac has poorer prognosis and doubled rates of pregnancy loss.
According to Cunningham et al and his colleagues who studied 40 pregnant with transvaginal ultrasonography between week 5-12 and observed that gestational sac of the cases which experienced miscarriage were smaller than normal, starting from week $5 .{ }^{13}$

In present study, it was concluded that pregnant women with large yolk sac had high incidence of abortion (78.57\%) followed by IUGR $(21.42 \%)$. Pregnant women with bradycardia also showed poor outcome showing incidence of Abortion, IUGR, PIH to be 38\%, 33.33\%, $23.80 \%$ respectively as compared to control group which showed only $7.7 \%$ pregnancy loss, $9.9 \%$ IUGR and $8.4 \%$ PIH.

According to Fotios et al, EHR and YSD progressively increase in healthy pregnancies during the first trimester. Embryonic bradycardia and absence of yolk sac or even a smaller yolk sac diameter than expected for any gestational age are predictors of poor pregnancy outcome during the first 12 weeks. ${ }^{14}$ In our study we found that patients with symptomatic subchorionic haemorrhage had more increased risk of complications like Spontaneous abortion, IUGR and preterm labor as compared to patients with subchorionic haematoma $<4 \mathrm{~cm}^{2}$.

According to Nagy et al, A retroplacental position of the hematoma was significantly correlated with an increased risk for adverse maternal and neonatal complications. The presence or absence of symptoms of threatened abortion did not affect these outcomes. The rates of pregnancyinduced hypertension (RR 2.1; CI 1.5, 2.9) and preeclampsia (RR 4.0; CI 2.4, 6.7), were significantly greater in the hematoma group.

Placental abruption (RR 5.6; CI 2.8, 11.1) and placental separation abnormalities (RR 3.2; CI 2.2, 4.7) were also 
significantly more frequent in the hematoma group. Perinatal complications, including the rate of preterm delivery (RR 2.3; CI 1.6, 3.2), fetal growth restriction (RR 2.4; CI 1.4, 4.1), fetal distress (RR 2.6; CI 1.9, 3.5), meconium-stained amniotic fluid (RR 2.2; CI 1.7, 2.9), and neonatal intensive care unit admission (RR 5.6; CI $4.1,7.6)$, were also significantly increased in this group. ${ }^{15}$

\section{CONCLUSION}

In today's time with availability of high end Sonography machines, Transvaginal sonography should be best utilized by not only looking at beating heart but also yolk sac, size of gestational sac, Heart rate because like uterine artery Doppler these markers are also predictors of complications to great extent especially in early pregnancy when uterine artery Doppler is not useful and can screen out cases which need close follow up. The accurate recognition of normal and abnormal sonographic findings concerning the yolk sac and heart rate can be used to anticipate the course of pregnancy. But still large case control studies are needed to establish the role of these markers. In pregnant patients with threatened abortion size of sub-chorionic haematoma can definitely predict the outcome of pregnancy and can be helpful in patient counselling.

Funding: No funding sources Conflict of interest: None declared

Ethical approval: The study was approved by the Institutional Ethics Committee

\section{REFERENCES}

1. Wilcox AJ, Weinberg CR, O'Connor JF. Incidence of early pregnancy loss. $\mathrm{N}$ Engl $\mathrm{J}$ Med. 1988;319:189-94.

2. Lindsay DJ, Lovett IS, Lyons EA. Yolk sac diameter and shape at endovaginal US: predictors of pregnancy outcome in the first trimester. Radiology. 1992;183:115-8.

3. Tan S, Pektaş MK, Arslan H. Sonographic Evaluation of the Yolk Sac. JIUM. 2012;31(1)87-95.

4. Hornberger LK, Sahn DJ. Rhythm abnormalities of the fetus. Heart. 2007;93(10):1294-300.

5. Varelas FK, Prapas NM, Liang RI, Prapas IM, Makedos GA. Yolk sac size and embryonic heart rate as prognostic factors of first trimester pregnancy outcome. Eur J Obstet Gynecol Reprod Biol. 2008;138:10-3.

6. Mackenzie WE, Holmes DS, Newton JR. Spontaneous abortion rate in ultrasonographically viable pregnancies. Obstet Gynecol. 1988;71:81-3.

7. Stefos TI, Lolis DE, Sotiriadis AJ, Ziakas GV. Embryonic heart rate in early pregnancy. J Clin Ultrasound. 1998;26:33-6.

8. Hyett J, Moscoso G, Nicolaides K. Abnormalities of the heart and great arteries in first trimester chromosomally abnormal fetuses. Am J Med Genet. 1997;69:207-16.

9. Elson J, Salim R, Tailor A, Banerjee S, Zosmer N, Jukovic D. Prediction of early pregnancy viability in the absence of an ultrasonically detectable embryo. Ultrasound Obstet Gynecol. 2003;21:57-61.

10. Nyberg DA, Mack LA, Harvey D, Wang K. Value of the yolk sac in evaluating early pregnancies. J Ultrasound Med. 1988;7:129.

11. Nyberg DA, Laing FC, Filly RA. Threatened abortion: sonographic distinction of normal and abnormal gestation sacs. Radiology. 1986;158:397400.

12. Ball RH. The sonography of pregnancy loss. Semin Reprod Med. 2000;18:351-5.

13. Cunningham FG, Gant NF, Leveno KJ, Gilstrap LC III, Health JC, Wenstrom KD. $21^{\text {st }}$ ed. Williams obstetrics. New York: McGraw-Hill; 2001:866-67.

14. Fotios K Varelas, Nicolaos M. Prapas, Ren-Ing Liang Loannis M. Prapas Georgios A. Makedos. Yolk sac size and embryonic heart rate as prognostic factors of first trimester pregnancy outcome. European J Obstetr Gynecol Reproduct Biol. 2008;138(1):10-3.

15. Nagy S, Bush M, Stone J, Lapinski RH, Gardo'S. Clinical significance of subchorionic and retroplacental hematomas detected in the first trimester of pregnancy. Obstet Gynecol. 2003;102(1):94-100.

Cite this article as: Bamniya $\mathrm{J}$, Panchal D, Singh $\mathrm{P}$, Shah A, Ladola H. Early sonographic markers and prediction of pregnancy outcome: a prospective study. Int J Reprod Contracept Obstet Gynecol 2017;6:2471-4. 